\title{
Controle Inteligente da Pressão Hidráulica com Ênfase na Eficiência Energética
}

\author{
Mikaele L. Diniz* Thommas K. S. Flores* \\ Juan M. Villanueva* Heber P. Gomes* \\ Pedro H. M. de Andrade* \\ * Universidade Federal da Paraíba, PB, (e-mail: \\ mikaele.diniz@cear.ufpb.br, thommas.flores@cear.ufpb.br, \\ jmauricio@cear.ufpb.br, heberp@uol.com.br, \\ pedro.andrade@cear.ufpb.br)
}

\begin{abstract}
The problem of water resources has become an urgent problem in recent years, from a hydropower or ecological point of view. Placing the focus on the water distribution networks, the indices of water losses occurring in the whole process stand out. These losses can reach values higher than $38 \%$, the main factor causes such losses is leaks due to excess pressure in the ducts that compose the system. As a solution, the use of pressure reducing valves and microgeneration devices in order to reduce the pressure in the ducts can be employed, but these methods insert losses in the system and the energy return is negligible. On the other hand, the use of frequency inverts to control the speed of rotation of the cluster as the network control techniques closes as an alternative for the improvement of energy efficiency. The water supply systems are nonlinear time-varying, the classical control methods may not present a good dynamic result. In view of this, this paper proposes a controller based on fuzzy logic to control hydraulic pressure of a water pumping system with an emphasis on the reduction of active electrical power used during this process, resulting in a maximum reduction of $40 \%$ of active electric power applied to the motor-pump assembly, besides the action of the controller maintain the constant pressure independent of the required demand, consequently reducing the actual losses due to leaks.

Resumo: O problema dos recursos hídricos tornou-se urgente nos últimos anos, seja do ponto de vista hidroenergético ou ecológico. Quando o foco são as redes de distribuição de água, os índices de perdas de água que ocorrem em todo processo se destacam. Essas perdas podem alcançar valores superiores a $38 \%$, o principal fator que ocasiona tais perdas é vazamentos por excesso de pressão nos ductos que compõe o sistema. Emprega-se o uso de válvulas redutoras de pressão e dispositivos de microgeração com a finalidade de diminuir a pressão nos ductos, porém estes métodos inserem perdas no sistema e o retorno energético é ínfimo. Por outro lado, o uso de inversores de frequência para o controle da velocidade de rotação do conjunto motobomba unido as técnicas de controle em malha fechada surge como uma alternativa para o aprimoramento da eficiência energética. Porém, como os sistemas de abastecimento de água são não lineares e variantes no tempo, os métodos de controle clássicos podem não apresentar um bom resultado dinâmico. Em vista disto, este artigo propõe um controlador baseado em lógica fuzzy para controle da pressão hidráulica de um sistema de bombeamento de água com ênfase na redução da potência elétrica ativa usada durante este processo, obtendo como resultado a redução máxima de $40 \%$ da potência elétrica ativa aplicada ao conjunto motobomba, além da presença do controlador manter a pressão constante independente da demanda requerida, consequentemente diminuindo as perdas reais decorrente de vazamentos.
\end{abstract}

Keywords: Hydraulic pressure control; Energy efficiency; Fuzzy control; Intelligent control; Pumping system.

Palavras-chaves: Lógica fuzzy; Controle da pressão; eficiência energética; Controle fuzzy; Controle inteligente, Sistema de bombeamento. 


\section{INTRODUÇÃO}

A escassez dos recursos hídricos tornou-se um problema urgente nos últimos anos, seja do ponto de vista hidroenergético ou ecológico, devido sua importância socioeconômica (Lezier et al. (2017)). Quando o foco são as redes de distribuição de água, o enfoque são as perdas que ocorrem em todo processo, desde a capitação até o usuário final, segundo SNIS (2018) estas perdas podem alcançar valores superiores a $38 \%$ e dependendo do município, esse percentual pode atingir $60 \%$ e em sua grande maioria e causada por perdas reais, isto é vazamento de água por excesso de pressão nos ductos que compõe o sistema (Gomes et all. (2012)).

Em contra partida, Creaco et all. (2017) propõe o uso de válvulas redutoras de pressão (VRP) como opção para obter valores ótimos de pressão ao longo da rede de distribuição de água, com o benefício de reduzir a quantidade de vazamento.

Porém, esta aplicação é energéticamente deficiente, pois este dispositivo insere perda de carga ao sistema de bombeameno, o qual estará sempre operando em velocidade nominal, isto é, será usado a máxima potência elétrica em um sistema sujeito a restrição da potência hidraulica, em forma de pressão e vazão, por meio de uma válvula redutora de pressão.

Ademais, outra alternativa para o controle de pressão em redes de abastecimento de água é o uso de dispositivos para microgeração localizados na descarga do sistema, isto é, através da instalação de uma turbina únida a um gerador, o sistema rezur a pressão e recupera a energia para abastecer a bomba. Porém, há fatores limitadores no uso deste método, como: a conveniência da instalação, baixa quantidade de energia gerada, o alto custo das turbinas tradicionais e a baixa eficiência (Fontana et all. (2016)).

Além disso, outro fator de suma importância nos sistemas de abastecimento de água é a eficiência enegética dos conjuntos motobombas, pois segundo Gomes et all. (2012) estes concentram aproximadamente $90 \%$ dos gastos com eletricidade das concessionárias de água.

Em vista disto, o uso de inversores de frequência para o controle da velocidade de rotação do conjunto motobomba (CMB) surge como uma forma de aumentar a eficiência energética dos sistemas de abastecimento de água. Porém a estratégia de controle adotada é a chave primordial no gerenciamento de energia e deve ser estabelecido por meio das características do sistema de bombeamento e a demanda requerida (Luo et all. (2015)).

Concomitantemente a isto, Wu et all. (2017) sugere o uso de um controle fuzzy-PD como uma alternativa frente aos controladores Proporcional Integral e Derivativo (PID), pois os sistemas de abastecimento de água apresentam características não lineares, variante e atraso no tempo, consequentemente o controlador PID, neste caso, não apresentam bom desempenho dinâmico.

Ademais, Barreiro-Gomez et all. (2017) propõe o uso do Controle Preditivo por Modelo (MPC), este tipo de controlador é capaz de lidar com mútiplos objetivos de controle, considerando-os dentro de uma função custo, que implica determinar uma prioridade para cada um dos objetivos, sendo necessário uma metodologia de ajuste dinâmico, que, neste caso, é usado a teoria dos jogos evolucionário. Porém este tipo de controlador exige um alto esforço computacional devido ao seu mecanismo de predição e ajuste.

Portanto, tendo em vista a dinâmica variável e alta complexidade no modelamento matemático das redes de bombeamento de água para a aplicação de controladores clássicos, este trabalho dedica-se a elaboração de um controlador fuzzy aplicado a regulação da velocidade do conjuto motobomba de um sistema de bombeamento.

O estudo contempla a análise da característica do controlador proposto quando submetido a pertubação, isto é, devido a variação da demanda. Além disso, realizou-se um estudo hidroenergético durante os ensaios, por meio da potência elétrica ativa que o inversor de frequência fornece ao conjunto motobomba.

Os ensaios experimentais foram realizados em uma das bancadas do Laboratório de Eficiência Energética e Hidráulica em Saneamento da Universidade Federal da Paraíba (LENHS/UFPB), o qual é capaz de emular uma rede de distribuição de água totalmente instrumentalizado e com as características necessárias para o estudo em questão.

\section{SISTEMAS DE ABASTECIMENTO DE ÁGUA}

Em geral, um sistema de abastecimento de água é composto por manancial (fonte que fornece a água), a captação e a estação elevatória; estação de tratamento; reservatório; rede de distribuição e ligações domiciliares (Gomes et all. (2012)). Na Fig. 1 está ilustrado um esquema hidráulico de um sistema de bombeamento. Dessa forma, a altura manométricatotal $(H)$ é o carregamento que deve ser superado pela energia fornecida pela bomba na forma de pressão e vazão.

Os sistemas de bombeamento de uma rede de distribuição de água, o CMB trabalha com o ponto de funcionamento móvel percorrendo a curva característica conforme a demanda do consumo (Gomes et all. (2012)). Como a demanda varia nesse sistema de distribuição de água, a pressão do sistema deve ser monitorada para evitar que em momentos de baixo consumo não se tenha elevados valores de pressão causando possíveis rompimentos nas tubulações e obtendo perdas de água e desperdício de energia.

Dessa forma, como as bombas são dispostivos que apresentam pontos de operação diferentes, então para sua correta seleção é necessário conhecer as características do sistema hidráulico o qual deseja-se instalar. Sendo assim, o ponto de operação da bomba é definido através da suas curvas características, isto é, gráfos que relaciona o altura manométrica e o volume bombeado e o rendimento da bomba (Gomes et all. (2012)).

A altura manométrica é o somatório de duas componentes: altura estática e altura dinâmica. A altura estática é a 


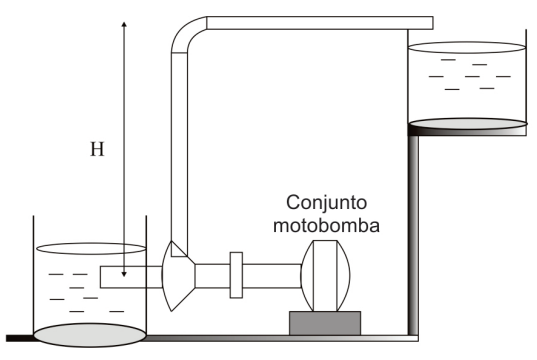

Figura 1. Esquema hidráulico de um sistema de bombeamento.

Figura 2. Curva característica da bomba.

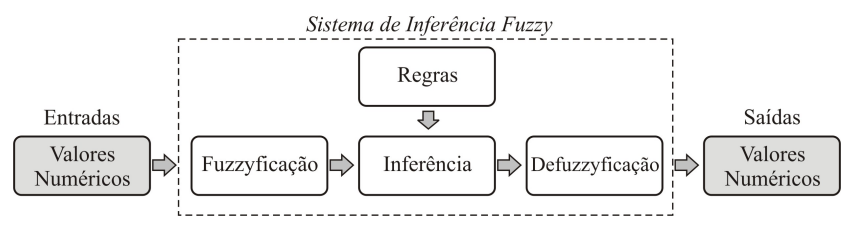

Figura 3. Fluxograma de um sistema baseado na lógica fuzzy

diferença entre o ponto de sucção e o ponto de ajuste, isto é, corresponde a uma altura geométrica. A altura dinâmica é a soma da carga de perdas apresentada em tubos e conexões, variando em relação ao quadrado do fluxo bombeado. Normalmente a altura manométrica recebe a unidade de metros de coluna de água (m.c.a.), descrito por $H$, conforme ilustrado na Fig. 1 (Gomes et all. (2012)).

Segundo Gomes et all. (2012) o gráfico $H \times Q$ da Fig. 2 ilustra a curva da bomba, este relacionando a altura manométrica $(H)$ para diferentes valores de vazão $(Q)$, mantendo-se a rotação da bomba constante. A curva " $A$ " foi obtida para cada valor de $H_{i}$, que corresponde ao valor de $Q_{i}$, em que o subscrito " $"$ " refere-se ao ponto do gráfico estudado. A curva " $B$ " pode ser obtida da mesma maneira, porém conside-se que para cada valor de vazão é acrescido às alturas referentes às perdas e a energia cinética remanescente, representados por $V^{2} / 2 g$ e $H_{f}$, respectivamente, em que $g$ é a gravidade e $V$ é a velocidade do fluido no ducto.

\section{FUNDAMENTOS DA LÓGICA FUZZY}

A lógica fuzzy (nebulosa) baseia-se no modo de raciocínio aproximado ao invés de exato, isto é, os valores verdade são expressos linguisticamente e podem assumir em uma proposição um subconjunto fuzzy de qualquer conjunto parcialmente ordenado em um intervalo unitário, diferente do conceito dos conjuntos clássicos, em que a proposição é um elmento de um conjunto finito, ou uma algebra booleana (Gomide et all. (1994)).

No diagrama da Fig. 3 estão inlustrados os principais blocos de um sistema baseado na lógica fuzzy, os quais segundo Hooda et all. (2016) estes blocos podem ser entendidos como sendo:

Os valores de entrada no sistema de inferência fuzzy são valores numéricos, assim é necessário um processo para a conversão de valores numéricos para conjuntos nebulosos. Esse processo é chamado de fuzzyficação, que

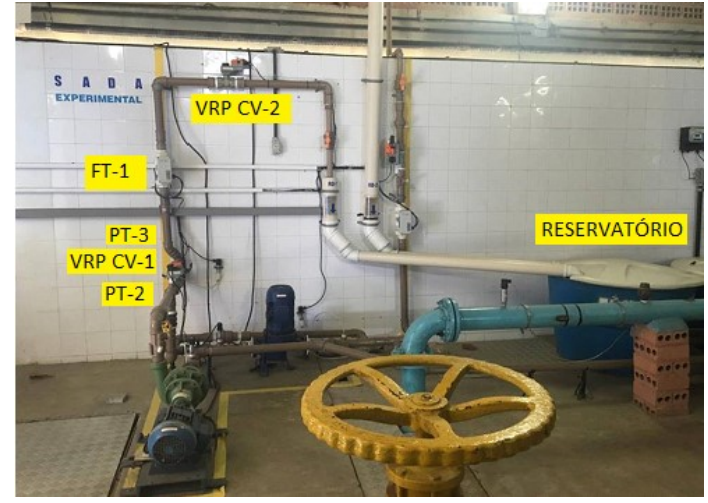

Figura 4. Bancada Experimental

torna possível que a máquina de inferência processe regras, em termos de variáveis linguísticas.

Em seguida, o processo de inferência avalia as regras do sistema (conhecimento), processando os valores dos antecedentes e consequentes sobre cada regra. As regras fuzzy coordenam a ação de tomada de decisão que este sistema deve tomar, a agregação dessas regras resulta em um conjunto fuzzy, que define a saída do controlador.

Por fim, os valores das variaveis linguísticas recebidas serão inferidas pelas regras difusas e convertido a um valor numérico por meio da defuzzyficação.

\section{BANCADA EXPERIMENTAL}

Na Fig. 4 se ilustra a bancada experimental utilizada neste trabalho, a qual encontra-se localizada no Laboratório de Eficiência Energética e Hidráulica em Saneamento na Universidade Federal da Paraíba (LENHS-UFPB). Esta bancada foi construída com o propósito de emular duas regiões com diferentes topologias, Zona Alta e outra para uma Zona Baixa. Ela é composta por um CMB de $3 \mathrm{CV}$, um booster (BST) de $3 \mathrm{CV}$, canos, dois reservatórios, transdutores de pressão do tipo piezoresistivo e vazão do tipo eletromagnético e válvulas redutoras proporcionais (VRP).

Como estudo de caso, foi utilizado apenas a zona baixa do sistema experimental. Esta região é compreendida pelo percurso, sem ramificação, desde a saída do CMB, e continua a percorrer pelo sensor de pressão PT-2, da VRP CV-1, do segundo sensor de pressão PT-3, pela segunda VRP CV-2 e finalmente, chegando ao reservatório.

\section{CONTROLE DE PRESSÃO USANDO LÓGICA FUZZY}

O sistema de controle proposto neste trabalho, tem por objetivo controlar a pressão no ponto PT-3 por meio de uma malha realimentada. Para projetar o controlador fuzzy foi necessário realizar a caracterização da planta sobre o qual o sistema de controle vai atuar. Para isso, foram construídas curvas sobre diferentes cenários dos principais elementos presentes no sistema experimental estudado: CMB, a válvula redutora de pressão CV-2 e a pressão do transdutor PT-3.

Portanto, os experimentos foram realizados variando o ângulo da válvula proporcional e a velocidade de rotação 


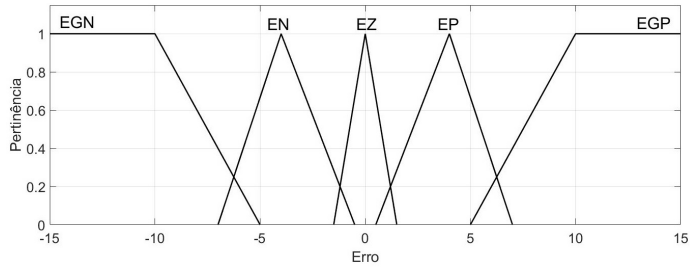

Figura 5. Funções de pertinência da variável Erro

do CMB por meio de um inversor de frequência. $\mathrm{O}$ ângulo da VRP pode variar de 0 a 90 graus, sendo 0 graus totalmente aberta e 90 graus totalmente fechada. Por fim, a velocidade de rotação do $\mathrm{CMB}$ variou-se entre 35 e 55 $\mathrm{Hz}$.

A elaboração das funções de pertinência das variáveis de entrada e saída, além das regras usadas na elaboração da lógica que compõe o controlador proposto baseouse no conhecimento do especialista após a analíse do comportameno da planta em diferentes cenários.

Pelas análises obtidas nos ensaios realizados, definiu-se que a pressão máxima fornecida pelo CMB foi de 9 m.c.a.. Por outro lado, ao estudar os limites da vazão, constatou-se que o sistema operando no máximo, isto é, a posição da válvula em 30 graus, o valor de pressão citado anteriormente permite uma vazão de $2,5 \mathrm{~m}^{3} / \mathrm{h}$ e para o menor consumo $0,5 \mathrm{~m}^{3} / \mathrm{h}$.

Para melhor entendimento, serão apresentadas separadamente cada uma das etapas do projeto do controlador fuzzy proposto, iniciando-se com as definições de entrada e saída, construção das funções de pertinência e criação das regras.

\subsection{Definição das variáveis de entrada de saída}

As variáveis de entrada do controlador correspondem a dados reais do sistema, proveniênte do transdutores de pressão (PT-3) e processados por uma placa de aquisição de dados, para que assim o controlador possa tomar decisões.

Dessa forma, determinou-se como entradas o erro da pressão, isto é, a diferença entre o valor da pressão desejada e da pressão medida e a variação do erro da pressão, respectivamente denominadas de: $E$ e $d E$.

Sendo assim, o conjunto da variável erro $(E)$ definiuse entre -15 e 15 m.c.a., corresponde a faixa da pressão observada durante os experimentos prelimináres. Portanto, esta faixa de operação está associada a cinco funções de pertinências como se ilustra na Fig. 5, cuja descrição é dada por: erro grande positivo (EGP), erro positivo (EP), erro zero (EZ), erro negativo (EN) e erro grande negativo (EGN).

Do mesmo modo, definiu-se as funções de pertinência da variável variação do erro da pressão $(d E)$, onde o domínio foi delimitado a um intervalo de -5 a 5 m.c.a. Sendo assim, foram foram definidas cinco funções de pertinência, como ilustrado na Fig. 6, cuja descrição é dada por: derivada do erro grande positiva (DEGP) que indica que a pressão está aumentando rapidamente; derivada do erro positivo (DEP) a pressão está aumentando; zero derivada da pressão (DEZ) indica que o valor está estabilizado; derivada do erro negativo (DEDN) a pressão está diminuindo não

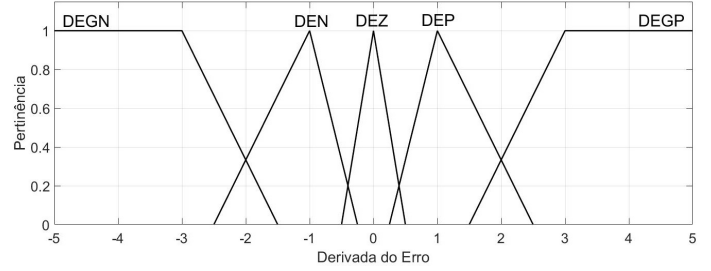

Figura 6. Funções de pertinência da variável variação do erro

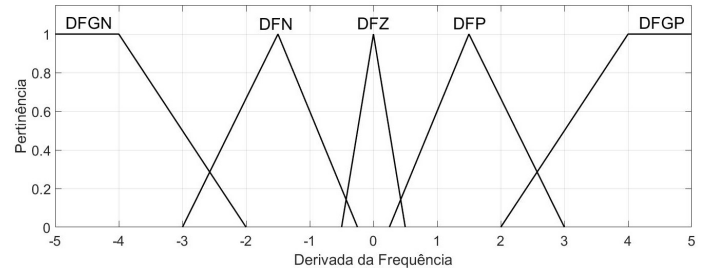

Figura 7. Funções de pertinência da variável variação da frequência

Tabela 1. Regras fuzzy.

\begin{tabular}{|c|c|c|c|c|c|c|}
\hline & \multicolumn{6}{|c|}{ Variação do erro } \\
\hline \multirow{6}{*}{$\begin{array}{l}\mathbf{E} \\
\mathbf{r} \\
\mathbf{r} \\
\mathbf{o}\end{array}$} & & DEGN & DEN & DEZ & DEP & DEGP \\
\hline & EGN & DEF & DFGN & DFGN & DFGN & DFGN \\
\hline & EN & $\overline{\mathrm{DF}}$ & $\overline{\mathrm{DFN}}$ & DFN & $\overline{\mathrm{DFN}}$ & DFGN \\
\hline & $\mathbf{E Z}$ & DFZ & DFZ & DFZ & DFZ & DFZ \\
\hline & DEP & DFGP & DFP & DFP & DFP & DFP \\
\hline & DEGP & DFGP & DFGP & DFGP & DFGP & DFP \\
\hline
\end{tabular}

tão rápido; derivada do erro grande negativo (DEGN) indica que a pressão está diminuindo rapidamente.

Por fim, para a variável de saída, isto é, a variação da frequência $(d F)$, definiu-se um domínio delimitado entre -5 a $5 \mathrm{~Hz}$. As variáveis linguísticas associadas a cada função de pertinência foram: DFGN (derivada da frequência grande negativo) fornece ao sistema um grande decremento a frequência; DFN (derivada da frequência negativo) fornece um decremento a frequência; DFZ (zero derivada da frequência zero) mantém atual igual a anterior; DFP (derivada da frequência positiva) fornece um incremento a frequência e DFGP (derivada da frequência grande positiva) fornece ao sistema um grande incremento a frequência. Na Fig. 7 ilustra-se o grafo destas funções.

\subsection{Construção das regras}

Sendo considerada a relação entre a pressão na saída do sistema que deseja-se controlar e a velocidade de rotação do conjunto motobomba, foram elaboradas 25 regras, com os seguintes critérios: erro em regime permanente menor que $5 \%$, tempo de subida menor que 10 segundos, tempo de assentamento menor que 20 segundos e sobressinal inferior a $10 \%$. Dessa forma, o conjunto das regras estão contidas na Tab. 1.

Por fim, considerou-se o processo de inferência do tipo Mamdani e o método do centro de gravidade da área para a etapa de de defuzzificação, afim de se obter resultado numérico da variável de saída. Hooda et all. (2016) explica toda a dedução matemática destes processos. 

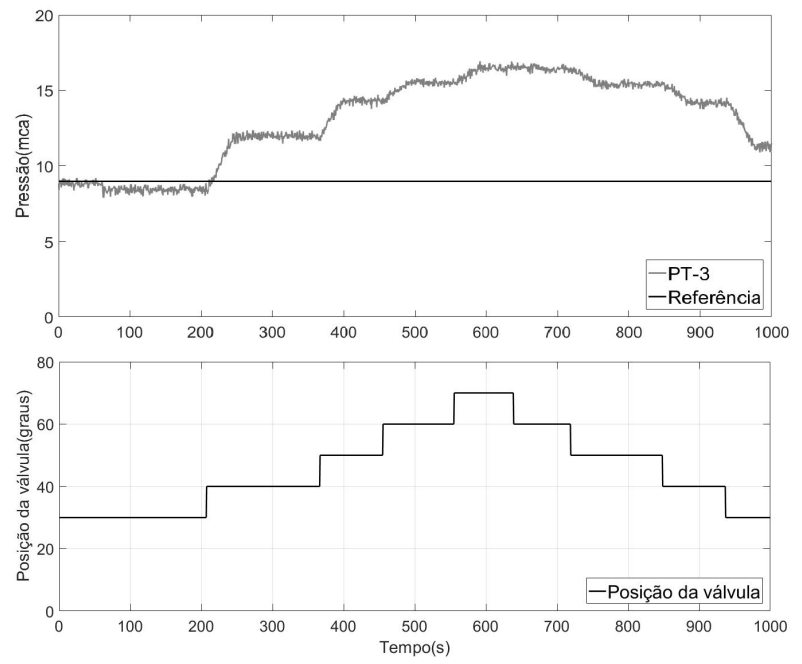

Figura 8. Operação em malha aberta com demanda variável.

\section{RESULTADOS E DISCUSSÕES}

Nesta seção, com a finalidade de analisar a ação do controlador e seu impacto na potência elétrica do sistema de bombeamento, realizou-se três experimentos distintos, conforme listado a seguir:

- Experimento I: Operação do sistema em malha aberta para o consumo variando entre o mínima e a máxima demanda;

- Experimento I: Validação do sistema operando com o controlador fuzzy;

- Experimento III: Operação do sistema em malha fechada sobre a ação do controlador proposto e sujeito a variação da demanda.

\subsection{Experimento $I$}

Este experimento teve como objetivo analisar a faixa de operação da pressão na saída do sistema sem a atuação do controlador mediante a variação da demanda, a qual foi emulada por meio da variação da abertura da VRP, com o CMB inicialmente o conjunto motobomba partido do repouso, isto é, velocidade de rotação igual a zero.

Na Fig. 8 nota-se que durante a demanda mínima, a pressão no ponto PT-3 se eleva, atingindo 12 m.c.a., um valor acima do necessário para atender com eficácia o consumidor final, que é 10 m.c.a.. Sendo assim, há um excesso de pressão durante a demanda é miníma, causando vazamento nos ductos e/ou rompimento dos mesmos e o aumento do consumo de energia elétrica.

\subsection{Experimento II}

O segundo experimento foi realizado com o intuito de observar as características da variável a ser controlada, isto é, a pressão na saída do sistema, quando está sujeita a ação do controlador proposto para uma entrada degrau. Para isto, foi utilizado um degrau de pressão que corresponde ao valor desejado com amplitude de 9 m.c.a., este valor de amplitude foi escolhido devido a característica construtiva do sistema, o qual não alcança 10 m.c.a, que seria o ideal,

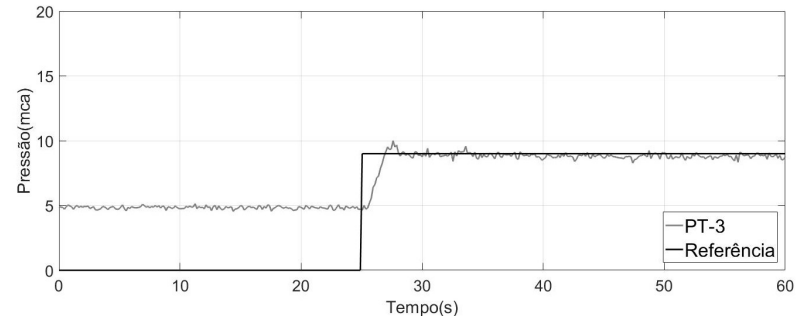

Figura 9. Resposta do sistema controlado.

Tabela 2. Característica da saída do sistema controlado

\begin{tabular}{|l|c|}
\hline \multicolumn{1}{|c|}{ Características } & Valor \\
\hline Sobressinal & $9,56 \%$ \\
\hline Tempo de assentamento & $10,4 \mathrm{~s}$ \\
\hline Tempo de subída & $5,6 \mathrm{~s}$ \\
\hline Erro em regime permanente & $1,77 \%$ \\
\hline
\end{tabular}

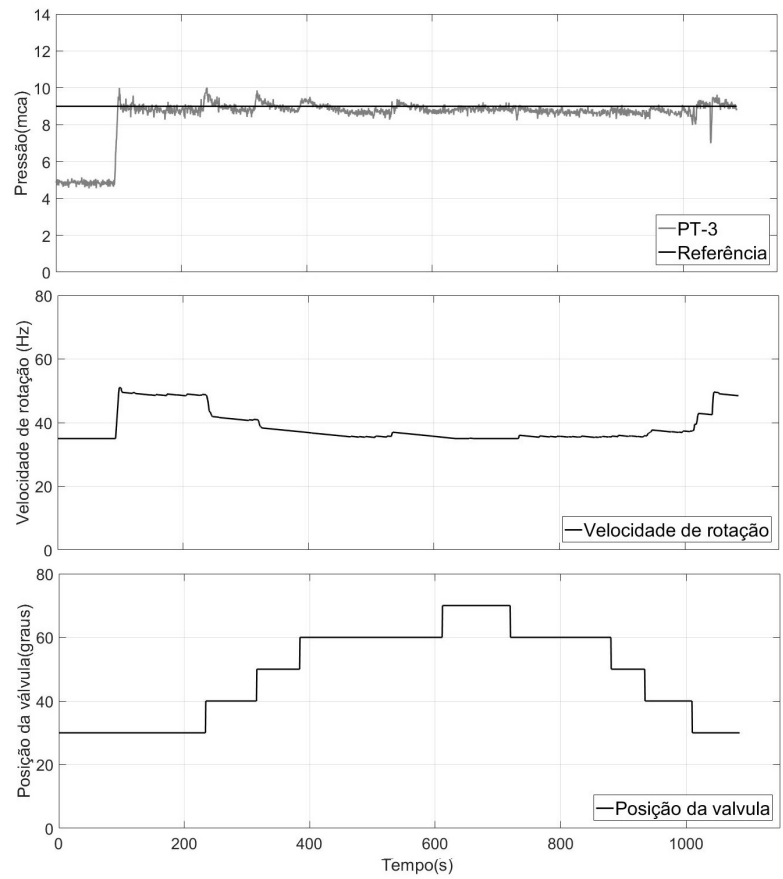

Figura 10. Resposta do sistema controlado com demanda variável.

quando a VRP está totalmente aberta. A resposta ao degrau é ilustrada na Fig. 9 e as características da resposta durante o regime permanente e no transitório estão na Tab. 2 .

\subsection{Experimento III}

Neste experimento, pretende-se avaliar a robustes da estrutura de controle baseado em lógica fuzzy proposta, quando o sistema está sujeito a variação da demanda requerida, isto é, emulada por meio da válvula proporcional CV-2. A metodologia proposta objetiva controlar a pressão no ponto onde está localizado o transdutor de pressão PT-3.

Na Fig. 10 está ilustado a resposta do sistema de controle para diferentes cenários de perturbação associada a demanda e emulado pela VRP CV-2. Observa-se que o sistema de controle modifica o valor da frequência do in- 


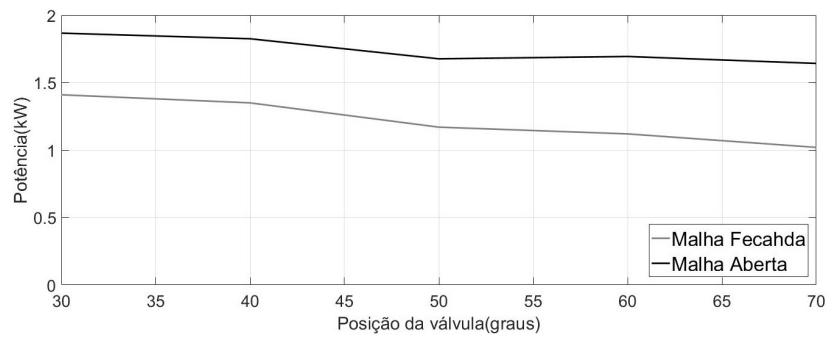

Figura 11. Potência elétrica ativa consumida durante os experimentos I e III.

versor, o qual atua para modificar a velocidade de rotação do conjunto motobomba.

Assim também, nota-se que o sistema de controle mantém o valor desejado da pressão no ponto PT-3 com um desempenho dentro da faixa proposta inicialmente durante seu projeto.

Portanto, a aplicação do controlador produziu uma demanda constante ao longo do período de consumo, consequentemente reduzindo a pressão durante a mínima demanda, isto é, o CMB que antes operava em velocidade nominal $(55 \mathrm{~Hz})$, agora opera com velocidade a baixo disto, diminuindo assim a energia necessária para fornecer ao usuário final a vazão e a pressão necessária para realização de suas atividades.

\subsection{Análise energética}

Para quantificar o ganho energético do sistema operando com e sem controlador, comparou-se os valores da potência elétrica ativa adquiridos por meio da interface homemmáquina durante o sistema operando em malha aberta (experimento I) e o sistema operando com o controlador fuzzy em malha fechada (experimento III).

A Fig. 11 ilustra o desempenho das medições da potência elétrica ao decorrer da variação da demanda, onde é possível observar uma redução de $20 \%$ para o cenário de máxima demanda (válvula em $30^{\circ}$ ) e de $40 \%$ para o cenário de menor demanda (válvula em $70^{\circ}$ ).

\section{CONCLUSÃO}

Em vista do que foi apresentado, o trabalho proposto buscou desenvolver uma metodologia para otimização dos setores de distribuição de água, permitindo o aumento da eficiência energética e melhorias da pressurização dos sistema de abastecimento de água.

Com relação ao controlador fuzzy desenvolvido, este atendeu as especificação desejadas, além de apresentar robustes, isto é, mesmo para diferentes cenários, ao qual o sistema foi submetido, manteve as características de projeto, com o erro em regime permanente igual a 1,77\% e sobressinal inferior a 10\%, conforme a Tab. 2 .

Do ponto de vista energético, a aplicação do controlador fuzzy unido a ação do inversor de frequência para diferentes valores de demanda, obteve-se uma redução de $40 \%$ da potência elétrica ativa usada pelo CMB durante o horário de mínima demanda e $20 \%$ durante o horário de máxima demanda.
Portanto, o uso de sistemas de controle inteligentes aplicado a redes de distribuição de água resulta no aumento da eficiência energética, devido a diminuição da energia elétrica gasta. Além da redução com custos de energia elétrica, o controle da pressão é um importante fator para evitar vazamentos, e assim desperdício da água potável. Portanto, causando um forte impactando econômico às concessionárias de água.

\section{AGRADECIMENTOS}

Os autores agradecem a Coordenação de Aperfeiçoamento de Pessoal de Nível Superior (CAPES), Laboratório de Eficiência Energética e Hidráulica em Saneamento (LENHS) e ao Grupo de Inteligência Computacional Aplicada (GICA) da Universidade Federal da Paraíba (UFPB) pelo suporte para a realização deste trabalho.

\section{REFERÊNCIAS}

Sistema Nacional de Informações sobre Saneamento (SNIS). Diagnóstico dos Serviços de Água e Esgotos2018. http://snis.gov.br., Acesso em: 09.05.2018. Ref.: IN049; FN002.

V. Lezier, M.Gusarova and A Kopytova Water supply of the population as a problem of energy efficiency on the example of the Tyumen region of Russia. In IOP Conference Series: Earth and Environmental Science, volume 90, n. 1, page 012069, 2017, October.

E. Creaco, A. Campisano, M. Franchini and C. Modica Unsteady flow modeling of pressure real-time control in water distribution networks. Journal of Water Resources Planning and Management, volume 143, n. 9, page 04017056, 2017.

N. Fontana, M. Giugni, L. Glielmo and G. Marini. Real time control of a prototype for pressure regulation and energy production in water distribution networks. Journal of Water Resources Planning and Managemen, volume 142, n. 7, page 04016015, 2016.

H. P. Gomes, P. D. Carvalho. Manual de Sistema de Bombeamento-Eficiência Energética. João Pessoa: Editora da UFPB, 2012.

Y. Luo, S. Yuan, H. Sun and Y. Guo. Energy-saving control model of inverter for centrifugal pump systems. Advances in Mechanical Engineering, volume 7, n. 7, page 1687814015589491, 2015.

J. Wu and M. Lu. Research of secondary constant pressure water supply system based on fuzzy-PID controller. In 2017 IEEE 3rd Information Technology and Mechatronics Engineering Conference (ITOEC), pages 462-465, 2017, October.

J. Barreiro-Gomez, C. Ocampo-Martinez and N. Quijano. Dynamical tuning for MPC using population games: A water supply network application. ISA transactions, volume 69, pages 175-186, 2017.

D. S. Hooda and V. Raich. Fuzzy logic models and fuzzy control: An introduction. Alpha Science International, 2016.

F. A. C. Gomide and R. R. Gudwin. Modelagem, controle, sistemas e lógica fuzzy. SBA controle $\&$ Automação, volume 4, n. 3, page 97-115, 1994. 